\section{Medicina social latinoamericana: aportes y desafíos}

\author{
Celia Iriart, ${ }^{1}$ Howard Waitzkin, ${ }^{1}$ \\ Jaime Breilh, ${ }^{2}$ Alfredo Estrada ${ }^{3}$ \\ y Emerson Elías Merhy ${ }^{4}$
}

Palabras clave: medicina social, América Latina, salud pública, difusión, Internet, base de datos.

\footnotetext{
1 Division of Community Medicine, Department of Family and Community Medicine, School of Medicine, University of New Mexico. La correspondencia debe enviarse a Celia Iriart a la siguiente dirección: Division of Community Medicine, Department of Family and Community Medicine, School of Medicine, University of New Mexico, 2400 Tucker Av. NE, Albuquerque, NM 87131, USA. Fax: 1-505-2724494. Correo electrónico: iriart@unm.edu

2 Programa EcoSalud, Centro de Estudios y Asesoría en Salud (CEAS), Quito, Ecuador.

3 Grupo de Investigación y Capacitación en Medicina Social (GICAMS), Santiago, Chile.

4 Departamento de Medicina Preventiva y Social, Facultad de Medicina, Universidad de Campinas, Campinas, São Paulo, Brasil.
}

La medicina social latinoamericana es un campo de conocimiento y práctica muy importante, pero poco conocido por quienes ejercen la medicina y la salud pública, y sobre todo por quienes no pueden acceder a la información escrita en español y portugués. Por este motivo, hemos desarrollado una investigación sobre este campo científico en la que, además de la revisión de 350 artículos, libros, capítulos, trabajos presentados en congresos e informes de investigación (publicados o no), escritos en español o en portugués desde la década de los setenta hasta la actualidad, hemos realizado 24 entrevistas en profundidad y 10 entrevistas informales con líderes y participantes activos de la medicina social.

Este artículo presenta el contexto en el que surgió la medicina social, las diferencias entre ella y la salud pública, sus teorías, métodos y debates, los principales temas o problemas considerados, y los desafíos que supone su difusión entre los anglohablantes y los profesionales de la medicina y la salud pública. Con anterioridad se han publicado dos artículos en inglés. El primero, publicado en The Lancet, presenta un revisión crítica del trabajo de los principales grupos de la medicina social en Argentina, Brasil, Chile, Colombia, Cuba, Ecuador y México (1). El otro, publicado en American Journal of Public Health, describe la historia del campo, los debates, los enfoques teóricos, metodológicos y técnicos, y los principales temas (2). La gran repercusión de estos dos artículos y el deseo manifestado por médicos y profesionales de la salud pública de América Latina de poder contar con este tipo de artículos en español nos mostró la importancia de difundir la medicina social también entre los hispanohablantes.

En el texto usaremos el nombre de medicina social, adoptado en la mayoría de los países latinoamericanos. Sin embargo, es oportuno aclarar que en Brasil la corriente ha adoptado el nombre de salud colectiva porque el movimiento sanitario surgido en ese país consideró importante destacar que sus análisis del conjunto de las prácticas y organizaciones de salud, incluida la práctica médica, abandonan la enfermedad y su tratamiento, y el acto médico, como eje central del proceso salud/enfermedad/atención. Asimismo, consideraron que había que resaltar la noción de que es un proceso construido colectivamente, tanto en la forma que adquiere en cada sociedad y momento histórico como en las posibilidades de transformarlo $(3,4)$. 


\section{CONTEXTO DE SURGIMIENTO DE LA MEDICINA SOCIAL}

La medicina social latinoamericana se desarrolla por la formación de grupos de académicos, practicantes e investigadores del campo de la salud que se unieron a los movimientos de trabajadores y de estudiantes y a las organizaciones populares disconformes con el modelo económico denominado desarrollista, que se implementó con intensidad en la década de los sesenta en América Latina. En su campo específico, los grupos ligados a la medicina social desarrollaron sus cuestionamientos en respuesta a la crisis de la salud pública, que se puso de manifiesto a fines de esa década. La salud pública también denominada desarrollista, que había sostenido que los efectos del crecimiento económico deberían llevar a un mejoramiento general de la salud, mostró sus limitaciones. Contrariamente a sus postulados, la observación de la realidad latinoamericana mostró un creciente deterioro de la salud pública, a pesar de que las economías latinoamericanas habían tenido un alto y sostenido crecimiento en las décadas anteriores. Los indicadores de salud, como la mortalidad infantil, entre otros, comenzaron a empeorar tras un período de cierta mejoría. En la mayoría de los países latinoamericanos, la mejoría de estos indicadores fue consecuencia de las transformaciones de las condiciones de vida y trabajo puestas en marcha por las modalidades latinoamericanas del estado del bienestar que precedieron a las propuestas económicas desarrollistas. El estado del bienestar amplió los derechos de los trabajadores y de los sectores sociales de menores ingresos, lo cual implicó una distribución más equitativa del ingreso a través de los salarios, y el acceso a la educación y a la salud financiado por el Estado a través de los impuestos o la seguridad social. El desarrollo de programas preventivos y de los sistemas de salud financiados con fondos públicos aumentó el acceso de la población al tratamiento de diferentes enfermedades $y$, al ser parte de un proceso redistributivo de la riqueza, facilitó la mejoría de los indicadores de salud.

Con el desarrollismo se revierte esta tendencia redistributiva de la riqueza. Mientras se observaba un crecimiento de los indicadores macroeconómicos en la mayoría de los países latinoamericanos, se deterioraban los indicadores sociales, entre ellos los de la salud (5-7). Hacia fines de los años sesenta, las transformaciones en la producción y en las condiciones de vida y trabajo implicaron un aumento de las enfermedades crónicas y degenerativas y de los accidentes de trabajo. Fue el comienzo de la coexistencia de enfermedades de la pobreza y de la riqueza. Las grandes desigualdades ante la enfermedad y la muerte se hicieron cada vez más vi- sibles. Las relaciones entre clase social y problemas de salud se hicieron evidentes, así como las inequidades en el acceso a los servicios de salud. La capacidad de diagnosticar y tratar enfermedades creció notablemente, pero el acceso de la población a estos avances fue muy diferente. Los gastos en salud crecieron, pero sus efectos en las condiciones de salud fueron muy limitados. Esta situación mostró que la existencia de más y mejores servicios no está relacionada con el mejoramiento de las condiciones de salud de la población, en especial cuando hay grandes diferencias en el acceso a estos avances (8-10).

En los años setenta se produce un notable crecimiento de la corriente de pensamiento de la medicina social, con el desarrollo de numerosos centros de investigación y docencia, y la aparición de revistas destinadas a publicar artículos de los autores más relevantes del campo. Juan César García fue un importante impulsor de ese crecimiento. Como coordinador de investigaciones de la Organización Panamericana de la Salud (OPS) en Washington, D.C., este médico argentino, formado en sociología en Chile, desarrolló desde 1966 hasta su muerte en 1984 importantes investigaciones y análisis sobre la educación médica, las ciencias sociales en medicina, los determinantes de clase social en los resultados de salud-enfermedad y las bases ideológicas de la discriminación contra los hispanos (11-13). García, junto con María Isabel Rodríguez, médica salvadoreña que vivió largos años en el exilio después de ser decana de la Facultad de Medicina de la Universidad de El Salvador, y Miguel Márquez, médico ecuatoriano, ambos funcionarios también de la OPS, dieron impulso a la medicina social latinoamericana no solo con sus trabajos, sino logrando el apoyo financiero de ese organismo para el desarrollo de investigaciones, reuniones científicas y organizativas de la corriente, publicación de libros y concesión de becas de formación. Las reuniones denominadas Cuenca I y II, realizadas en Ecuador y coordinadas por Márquez, marcaron el rumbo del movimiento de la medicina social. El apoyo financiero de la OPS fue importante para establecer y consolidar uno de los primeros programas de posgrado que se creó con esta perspectiva: la Maestría de Medicina Social de la Universidad Autónoma Metropolitana Xochimilco, de la Ciudad de México.

Cabe resaltar asimismo que en el proceso de afianzamiento y difusión de esta corriente de pensamiento tuvieron también destacada participación organizaciones no gubernamentales como el Centro de Estudios y Asesoramiento en Salud (CEAS) de Ecuador, el Grupo de Investigación y Capacitación en Medicina Social (GICAMS) de Chile y el Centro de Estudios Sanitarios y Sociales (CESS) de Argentina, entre otros. El CEAS sigue cumpliendo un 
papel fundamental en la difusión de la corriente a través de un esfuerzo editorial sostenido por el cual ha publicado numerosos libros de los más importantes pensadores de la medicina social. El GICAMS, por su parte, publicó durante muchos años una importante revista: Salud y Cambio. El CESS publica desde hace 25 años una revista que reúne artículos de importantes trabajos de la medicina social. Estas organizaciones capacitan profesionales y trabajadores de la salud, y participan activamente en el desarrollo de importantes investigaciones.

En Brasil, Colombia, Cuba y Venezuela, los cursos de formación, maestrías y doctorados se han realizado principalmente desde universidades públicas, lo cual ha permitido que la formación en medicina social se haya extendido considerablemente y que los profesionales así formados estén insertados en distintos niveles del sistema sanitario. La producción científica brasileña es en la actualidad una de las más importantes e innovadoras de América Latina. En Brasil, el esfuerzo de difusión a través de revistas científicas y libros es muy importante y se realiza desde ámbitos universitarios y organizaciones gubernamentales y no gubernamentales, tales como la Associação Brasileira de Saúde Coletiva (ABRASCO) o el Centro Brasileiro de Estudos em Saúde (CEBES). La cooperación entre diferentes organizaciones latinoamericanas posibilitó la creación de una red de intercambio y apoyo que permitió a muchos profesionales de la salud consolidar su formación, intercambiar experiencias con colegas de otros países y actuar como multiplicadores de este pensamiento. En los últimos años se han desarrollado numerosas investigaciones en colaboración con diversos países latinoamericanos, e incluso con los EE. UU.

\section{ENFOQUES TEÓRICO-METODOLÓGICOS: DIFERENCIAS CON LA SALUD PÚBLICA}

La medicina social latinoamericana se ha desarrollado dentro de un amplio y rico espectro de posiciones teóricas y metodológicas. Desde el origen de esta corriente se han llevado a cabo intensos debates sobre teorías, métodos y estrategias de cambio que todavía continúan, lo que demuestra la vitalidad de este campo de pensamiento y práctica (14). Los debates metodológicos toman en consideración el contraste entre los modelos empírico-funcionalistas e histórico-analíticos, y entre los enfoques macropolíticos y micropolíticos, así como el equilibrio entre las metodologías de investigación cualitativas y cuantitativas, y entre lo individual, lo grupal y lo colectivo como unidades de análisis (15-17).

El enfoque teórico-metodológico de los colectivos que estudia la medicina social le confiere a su praxis una característica muy diferencial con respecto a la salud pública. Esta considera como unidad de análisis a la población, a la que define como una suma de individuos que pueden ser clasificados en grupos según ciertas características (sexo, edad, educación, ingreso, raza o etnia). A manera de ejemplo, podemos señalar que en la epidemiología clásica los porcentajes de una población específica se calculan aritméticamente a partir de las características individuales de quienes componen ese grupo poblacional, seleccionadas a priori por el investigador para definir el grupo analizado. En cambio, los trabajos de la medicina social consideran a la población y a las instituciones sociales como totalidades cuyas características trascienden las de los individuos que las componen (15-20). Por lo tanto, la medicina social define los problemas y desarrolla sus investigaciones a través de unidades de análisis sociales e individuales, pero con un encuadre teórico-metodológico colectivo. Es decir, las especificidades individuales y grupales son analizadas en el contexto social que las determina. En este sentido, las principales categorías analíticas son la reproducción social, la clase social, la producción económica, la cultura, la etnia y el género, entre otras (21). Es a partir de estas categorías que las especificidades individuales, tales como el sexo, edad, educación, ingreso o raza, encuentran su determinación explicativa. Por ejemplo, si no se explicitan las características económicas, sociales, políticas e ideológicas específicas que, en una determinada sociedad y momento histórico, definen el ser mujer $\mathrm{u}$ hombre en relación con el problema estudiado, se están desconociendo los determinantes que subyacen a la clasificación por sexo (22). Lo destacable es que la definición del colectivo no se realiza a priori, sino que está ligada al problema que se pretende analizar. Lo anterior implica dar centralidad y hacer explícita la teoría en todo el proceso de investigación o de intervención en torno a un problema. Desde la medicina social se argumenta que la falta de explicitación de los supuestos teóricos en la medicina y la salud pública anglosajonas de corte empírico no significa ausencia de teoría. Por el contrario, una postura ateórica o antiteórica significa que la teoría fundamental está implícita y que muchas veces la desconocen los propios investigadores $(23,24)$.

En lo que se refiere a la relación entre la teoría y la práctica, desde la perspectiva de la medicina social se usa el concepto de praxis, entendida como la interrelación entre pensamiento y acción. En este sentido, los líderes de la medicina social, influenciados por el pensamiento de Gramsci, destacan el doble camino de la teoría que contribuye a los esfuerzos tendientes al cambio social, pero se nutre, a la vez, de estos esfuerzos (25). A menudo, las actividades de investigación se desarrollan en conjunto 
con sindicatos, grupos de mujeres, coaliciones indígenas y organizaciones comunitarias (26).

Otras diferencias entre la medicina social y la salud pública están dadas por el carácter estático o dinámico de la salud-enfermedad y el efecto del contexto social. La medicina social conceptualiza la salud-enfermedad como un proceso dialéctico y no como una categoría dicotómica. La epidemiología crítica estudia el proceso salud-enfermedad en el contexto social, considerando los efectos de los cambios de las condiciones sociales a lo largo del tiempo. El perfil epidemiológico de un colectivo social o institucional en una determinada sociedad requiere un análisis de múltiples niveles para comprender por qué y cómo las condiciones sociales, tales como la reproducción social, la producción económica, la cultura, la marginalización y la participación política, se organizan históricamente en distintos modos de vida característicos de los grupos situados en diferentes posiciones dentro de la estructura de poder, y determinan un acceso diferencial a condiciones favorables o protectoras, o a condiciones desfavorables o destructivas, estableciendo la dinámica del proceso salud-enfermedad $(15,27,28)$. Desde esta perspectiva teórica, en la salud pública, los modelos formales multivariados, tales como los modelos de regresión logística con la enfermedad como variable dependiente, dicotomizada según su presencia o ausencia, oscurecen la dialéctica del proceso salud-enfermedad $(17,19)$.

La teoría de la salud-enfermedad como un proceso dialéctico ha generado críticas de los enfoques tradicionales basados en la inferencia causal en medicina y salud pública $(8,15,19)$. En un nivel básico, los pensadores de la medicina social han criticado el modelo monocausal de explicación de la enfermedad. Con una perspectiva similar a la desarrollada por la medicina social europea del siglo XIX, los investigadores latinoamericanos mantienen la perspectiva de que las explicaciones simplistas por las cuales un agente específico es la causa necesaria y suficiente para producir una enfermedad no consideran adecuadamente las condiciones sociales que son determinantes para el desarrollo de la enfermedad $(29,30)$. Incluso los modelos multicausales, como los ecológicos, que consideran la interacción entre agente, húesped y ambiente, $\mathrm{o}$, más recientemente, los modelos de complemento causal e interacción biológica, definen la enfermedad de una manera relativamente estática. Las críticas desde el punto de vista de la medicina social han argumentado que, por dicotomizar la presencia o ausencia de una enfermedad, los modelos multicausales tradicionales no consideran adecuadamente los vínculos dinámicos por los cuales la dialéctica del proceso salud-enfermedad se ve afectada por las condiciones sociales. Estos análisis han su- gerido un enfoque más complejo de la causalidad, en el cual las condiciones sociales e históricas son consideradas como determinantes estructurales, es decir, que existen antes del problema analizado, y su comprensión permite especificar las dimensiones del mismo. Los determinantes sociales e históricos no son variables cuya única diferencia es el peso que el investigador les atribuye en la cadena causal.

Anticipando las tendencias metodológicas actuales en los EE. UU., los líderes de la medicina social latinoamericana han abogado desde mediados de los años setenta por un enfoque multimetodológico que "triangula" complementariamente métodos de análisis del nivel individual y social. Aun en las investigaciones iniciales, los investigadores mexicanos y ecuatorianos combinaron métodos y técnicas de recolección de información cuantitativos y análisis multivariado, con métodos cualitativos. Una de estas técnicas que ha mostrado capacidad para incrementar la calidad y cantidad de la información obtenida es la entrevista colectiva (18).

Pero es fundamental volver a señalar que para la medicina social el punto de partida es un replanteamiento teórico del problema, a la luz del cual los métodos y técnicas se subordinan a una lógica analítica de procesos dinámicos considerados en conjunto, y no a la inversa; es decir, no se subordinan a la preeminencia de una lógica empírica que asocia, a través de la metodología y de las técnicas, mayoritariamente cuantitativas o cualitativas cuantificadas, fragmentos de la realidad.

Aunque la teoría marxista ha estimulado los desarrollos de la medicina social, el trabajo conceptual se ha centrado en analizar las limitaciones de la teoría marxista más tradicional en el contexto latinoamericano y rescatar los elementos conceptuales que pueden contribuir a una comprensión contextualizada del proceso salud-enfermedad (31). Un concepto central que la medicina social rescata de la teoría marxista es el de clase social, definido por las relaciones de producción económica. En la teoría marxista, la característica más importante de la definición de clase social involucra la propiedad y el control de los medios de producción y, por ende, del proceso productivo. En la medicina social se argumenta que la explotación del trabajo y del desempleo mediante la dominación de los ocupados y de los desocupados es una condición inseparable de la producción económica. Como consecuencia de esta concepción, se mantiene una visión de la clase social que tiene sus raíces en el proceso de producción y no en características demográficas como los ingresos, la educación o el prestigio ocupacional, que se usan para realizar una estratificación social, pero dejan intocadas las relaciones que determinan los problemas sociales como la salud-enfermedad. Esta posición teórica relacionada con la producción 
económica ha guiado la elección de los problemas de investigación sobre el proceso de trabajo, tanto industrial como agrícola. Los grupos de la medicina social en Brasil, Chile, Ecuador y México han realizado estudios del impacto de la organización jerárquica del trabajo, de los procesos de producción y de las condiciones de trabajo sobre la salud mental y física (32-34).

Otro concepto importante para la medicina social es el de reproducción económica. La teoría marxista cuestiona la reproducción de las relaciones de explotación que, a través de generaciones, produce el sistema capitalista. Una de las instituciones más importantes que favorecen esta reproducción es la familia, especialmente mediante las relaciones de género, según el papel que se le asigna socialmente a cada integrante de la misma. Marx y Engels argumentaron, por ejemplo, que la explotación de los trabajadores estuvo intrínsicamente relacionada con la explotación de la mujer desde que la producción económica requirió la reproducción de la fuerza de trabajo, principalmente mediante las actividades de las mujeres en el ámbito familiar (35). En las sociedades contemporáneas, las mujeres a menudo soportan la "triple carga" del trabajo asalariado, el trabajo doméstico y el cuidado de los hijos. Por esta razón, los grupos de la medicina social de algunos países han colaborado en investigaciones que se centran en las mujeres trabajadoras y los efectos de su papel en la producción y reproducción económica (36).

La ideología es un tercer eje teórico de la medicina social. Comprende las ideas específicas y doctrinas de un grupo social. Una ideología "hegemónica" tiende a justificar los intereses de las clases que dominan una sociedad en un período histórico. La desmistificación de esta ideología es parte de la tarea teórica y política de la medicina social latinoamericana $(37,38)$. Durante los primeros años, el trabajo de desmistificación se centró en las políticas desarrollistas fomentadas por los gobiernos de EE. UU. y Europa $(8,10,24,28)$. Más recientemente, los esfuerzos de desmistificación se han centrado en las políticas del Banco Mundial y de otros organismos multilaterales de crédito que han alentado el crecimiento de la deuda, las privatizaciones y los recortes de los servicios públicos, basándose en los principios macroeconómicos de la libertad de mercado (39-41).

Las teorías europeas contemporáneas también han influenciado a la medicina social latinoamericana. Por ejemplo, los esfuerzos teóricos realizados en Italia sobre el proceso de trabajo han contribuido al enfoque conceptual que el grupo mexicano de la medicina social ha desarrollado con los sindicatos industriales $(18,32)$. El psicoanálisis, el análisis institucional y desarrollos filosóficos fran- ceses recientes han influenciado los esfuerzos de los investigadores argentinos y brasileros en sus estudios sobre los servicios de salud y la crítica de las ideologías en las políticas de salud, así como en la realización de propuestas de cambio (42-43).

\section{PRINCIPALES TEMAS Y SU RELACIÓN CON LOS MOVIMIENTOS SOCIALES Y POLÍTICOS}

Los grupos de la medicina social de diferentes países latinoamericanos han enfatizado los efectos de las políticas internacionales. Históricamente, tales trabajos han analizado el impacto del imperialismo económico, la extracción de materia prima y la explotación de la mano de obra barata. Más recientemente, se han centrado en las políticas macroeconómicas y el poder político de las corporaciones multinacionales y las agencias multilaterales de crédito. La carga de la deuda externa en los países del tercer mundo ha surgido como una importante preocupación. Los recortes presupuestarios, la privatización del sector público y la apertura de los mercados de la atención de la salud a las corporaciones multinacionales han recibido gran atención. Algunos grupos han colaborado en la evaluación de la atención gerenciada como una iniciativa de privatización de las corporaciones multinacionales y las agencias multilaterales de crédito. Estos estudios han destacado tanto los efectos perjudiciales en el acceso a los servicios de salud como el deterioro de la red de servicios de atención de la salud y han desmistificado las tesis de que las prácticas orientadas por el mercado mejoran las condiciones de los pobres (44-48).

Los grupos de la medicina social han vinculado sus investigaciones sobre políticas con esfuerzos organizativos que apuntan a cambiar las relaciones de poder. Estas acciones tratan de ampliar el debate público y reorientar las iniciativas de reforma hacia el encuentro con las necesidades de las poblaciones vulnerables. El grupo de la Universidad de Campinas en Brasil, nucleado en el Laboratório de Planejamento em Saúde, ha desarrollado importantes investigaciones sobre la micropolítica de los procesos de trabajo en las instituciones de salud y ha realizado múltiples intervenciones junto a los servicios de salud para modificar la relación entre las instituciones sanitarias y los usuarios. Para esto ha utilizado metodologías y técnicas innovadoras de enfoque institucional y de análisis de los procesos de trabajo (49-52). El grupo de Argentina nucleado en la Central de Trabajadores Argentinos colabora con los movimientos sociales y de trabajadores en la realización de análisis y propuestas alternativas a la privatización en salud (53). 
Varios grupos han sido pioneros en la investigación de los determinantes socioeconómicos. En Ecuador, algunos investigadores han tomado como eje la ecología urbana, los cambios económicos provocados por la producción petrolera y las relaciones de la estructura de inequidad de clase, género y etnia con la salud, así como los procesos de trabajo, para explicar los patrones de morbilidad y mortalidad (54-56). Estos investigadores ponen sus investigaciones al servicio del mejoramiento de las condiciones de vida y trabajo y del apoyo a las luchas de las coaliciones indígenas y los movimientos sociales.

Los investigadores mexicanos han trabajado con los sindicatos de la industria y las comunidades locales para esclarecer los problemas de salud física y mental que se derivan de los procesos de trabajo y del medio ambiente. En estos esfuerzos, han sido pioneros en el uso de métodos como la entrevista colectiva $(18,32,57)$. En Chile, el grupo de la medicina social ha llevado adelante investigaciones que vinculan las condiciones de género, trabajo y medio ambiente (58).

Las investigaciones sobre violencia y traumas psíquicos han recibido atención prioritaria en varios países. El interés por este tema refleja la historia de la mayoría de los países latinoamericanos, sufrida incluso por los miembros de la medicina social. En Colombia, la tradición social de violencia -vinculada antes a la pobreza y a los ciclos de rebelión, y más recientemente al tráfico de drogas y a las operaciones paramilitares- ha generado investigaciones sobre los efectos de la violencia en la salud $(59,60)$. En Chile se ha trabajado junto con organizaciones de derechos humanos para apoyar a las familias cuyos miembros han sufrido torturas, exilio o muerte durante la dictadura (61). En Argentina también se ha trabajado con los sobrevivientes de la represión - tanto víctimas directas como familiares - llevada a cabo por la última dictadura militar (1976-1983). Estos investigadores fueron influenciados por los estudios psicológicos sobre la violencia realizados en El Salvador por Ignacio Martín-Baró, un psicólogo salvadoreño formado en EE. UU., que fue asesinado en su país por fuerzas paramilitares (62-64).

\section{EL DESAFÍO DE SU DIFUSIÓN}

Este productivo campo de investigación, reflexión teórica y práctica requiere que se hagan importantes esfuerzos para difundirlo. Por un lado, se debe buscar aumentar su difusión entre los profesionales y trabajadores de la salud que no lo conocen, ya sea por problemas de idioma, o por su ausencia en las publicaciones médicas y de salud pública más tradicionales y de mayor circulación entre la mayoría de los profesionales de la salud. Por otro lado, esta difusión debe contribuir al intercambio entre la salud pública y la medicina social, permitiendo el enriquecimiento de ambas. Para eso estamos desarrollando en la Biblioteca del Centro de Ciencias de la Salud e Informática (Health Sciences Library and Informatics Center: HSL\&IC) de la Universidad de Nuevo México, EE. UU., un portal en Internet y una base de datos con resúmenes estructurados de libros, capítulos de libros, y artículos relevantes de la medicina social (http://hsc. unm.edu/lasm). La elección de los resúmenes estructurados se hizo siguiendo las recomendaciones del Grupo de Trabajo Ad Hoc para la Valoración Crítica de la Literatura Médica (Ad Hoc Working Group for Critical Appraisal of the Medical Literature), que desarrolló el concepto de resumen estructurado para ayudar a los lectores de materiales clínicos a seleccionar más rápidamente y con mayor precisión los artículos más apropiados, y para facilitar la revisión por pares de los artículos sometidos a arbitraje (65).

El material a ser colocado en el portal de la red es seleccionado con la colaboración de un $\mathrm{Co}^{-}$ mité de Expertos formado por líderes de la medicina social latinoamericana (la lista de los miembros del Comité puede consultarse en el portal). Los resúmenes estructurados se introducen en el portal y en la base de datos en tres idiomas: portugués, español e inglés. Los textos originales de donde se extraen los resúmenes están almacenados en la HSL\&IC, al igual que las 12 revistas latinoamericanas que, por su énfasis en la medicina social, se han suscrito en este proyecto con el asesoramiento del citado Comité. ${ }^{5}$ Otro aspecto del proyecto es la publicación electrónica en el portal, de forma experimental, de dos importantes revistas de la medicina social latinoamericana (Saúde em Debate, de Brasil, y una publicada en español que aún no se ha definido). Apoyándonos en la tecnología de Internet esperamos ampliar el número de suscriptores, tanto de EE. UU. como de otros países del mundo, para estas importantes revistas cuyos editores muchas veces se encuentran con problemas de financiamiento para su publicación y distribución en las formas tradicionales.

Un desafío central en este proyecto es la traducción al inglés de los textos de la medicina social latinoamericana, porque sus conceptos y desarrollos teóricos y metodológicos no encuentran su co-

\footnotetext{
5 Las revistas suscritas son: Cuadernos Médico Sociales y Salud Problema y Debate, de Argentina; Interface, Cadernos de Saúde Pública, Revista de Saúde Pública, Saúde e Sociedade, Saúde em Debate, Ciência e Saúde Coletiva y Revista Brasileira de Epidemiologia, de Brasil; Revista Cubana de Medicina Tropical y Revista Cubana de Salud Pública, de Cuba, y Salud Problema, de México.
} 
rrelato en la terminología corriente de la salud pública norteamericana. Muchas veces, obtener una traducción comprensible para los lectores del campo de la medicina o de la salud pública de habla inglesa dificulta la transmisión exacta, en toda su complejidad, de los conceptos de la medicina social. Sin embargo, consideramos que este primer esfuerzo abrirá las posibilidades futuras de profundizar en el conocimiento de este importante campo científico y permitirá avanzar en la precisión conceptual y en el intercambio con los investigadores y estudiosos de la medicina social latinoamericana.

\section{CONCLUSIÓN}

La medicina social latinoamericana ha surgido como un campo desafiante, por los aportes que ha hecho a la comprensión de los determinantes del proceso salud-enfermedad-atención y a su complejidad, así como por el uso de teorías, métodos y técnicas escasamente conocidos por la salud pública. El eje en los orígenes sociales de la enfermedad y la muerte temprana desafía las relaciones de poder económico y político en las sociedades. Los temas y hallazgos han llegado a ser pertinentes para problemas médicos y de salud pública en todo el mundo, y en especial para el análisis y la propuesta de alternativas a la inequidad en salud, la reforma de los sistemas sanitarios, los estudios de género, el estudio de las micropolíticas y macropolíticas que afectan a la atención sanitaria y a la salud de los trabajadores, entre otros. Los profesionales de la medicina social latinoamericana han usado teorías y métodos que distinguen sus esfuerzos de los de la salud pública. En particular, el eje en el contexto social e histórico de los problemas de salud, el énfasis en la producción económica y en la determinación social, y la vinculación de la investigación y la formación con la práctica política, han proporcionado enfoques innovadores para algunos de los problemas más importantes de nuestra época.

Las barreras de idioma y posiblemente el escepticismo acerca de las investigaciones que provienen del "Tercer Mundo" han favorecido el desconocimiento de esta importante corriente entre los profesionales sanitarios de los países del "Primer Mundo". Asimismo, el lenguaje y los métodos tradicionales de circulación de las revistas latinoamericanas de medicina social han sido un obstáculo para que accedan a esta importante producción científica muchos profesionales sanitarios de todo el mundo, cuyos trabajos podrían beneficiarse de los avances de la medicina social si esta estuviera más difundida y accesible. Para los EE. UU. y para otros países, la producción de la medicina social latinoamericana puede llegar a ser algo sumamente valioso a tener en cuenta, en especial en esta era de globalización, con sus peligros para la salud pública.

Agradecimientos. Este trabajo fue financiado parcialmente por subsidios de la Comisión Fulbright (Senior Fellowship for Independent Research, American Republics Program), del Centro Internacional Forgarty de los Institutos Nacionales de la Salud (National Institutes of Health) de los EE. UU. (TW 01982), de la Universidad de California (Pacific Rim Program), del Colegio Americano de Médicos (American College of Physicians - George Griffith Traveling Felowship), de la Facultad de Medicina de la Universidad de Nuevo México (Dedicated Health Research Funds) y de la Biblioteca Nacional de Medicina de los EE. UU. (National Library of Medicine - 1G08 LM06688).

En la primera etapa de la investigación sobre la medicina social (1994-2000) participó, además de los autores, Silvia Lamadrid, del Grupo de Investigación y Capacitación en Medicina Social (GICAMS) de Chile, quien ayudó en la recolección, análisis e interpretación de datos. En el proyecto de creación del portal en Internet y de la base de datos participan Holly Buchanan (co-investigadora principal), Jon Eldredge, Russ Davidson, Ruth Morris, Kevin Wiley y Jonathan Tregear, de la Universidad de Nuevo México. Emerson Merhy, Alfredo Estrada, Jaime Breilh y Norma Fernández son los investigadores principales de los equipos de Brasil, Chile, Ecuador y Argentina, respectivamente. Howard Waitzkin es el investigador principal de ambos proyectos, y Celia Iriart es la coordinadora general del proyecto de creación del portal en Internet y de la base de datos, así como de la publicación electrónica de la revistas latinoamericanas, además de investigadora asociada de la primera etapa.

A todos los colegas que participan en el proyecto de difusión, incluidos los miembros del Comité de Selección de Pares cuyos nombres están en el portal, agradecemos su dedicación e interés en ayudarnos a difundir la medicina social latinoamericana. Expresamos nuestra especial gratitud a numerosos colegas y amigos que nos ofrecen su constante asesoramiento, que participaron en las entrevistas y que son un ejemplo de coraje y compromiso con el desarrollo de la medicina social.

\section{SYNOPSIS}

\section{Latin American social medicine: contributions and challenges}

This piece presents and analyzes a number of issues related to social medicine: the context of the emergence of social me- 
dicine; the differences between social medicine and public health; the theories, methods, and debates in social medicine; the main subjects or problems considered in social medicine; and the difficulties of disseminating the concepts of social medicine among English-speaking persons and among medical and public health professionals in general. Latin American social medicine has challenged other views by contributing to an understanding of the determinants of the healthdisease-health care process and by using theories, methods, and techniques that are little known in the field of public health. Introducing Latin American social medicine, especially among English speakers, will be difficult due to the concep- tual complexity of this field for persons who are accustomed to the theoretical framework of public health and medicine and also due to skepticism concerning research coming from the Third World. A multidisciplinary team is facing this challenge through two primary initiatives: 1) the creation of an Internet portal and database where there are structured abstracts in English, Portuguese, and Spanish of books, book chapters, and articles on social medicine and 2) the electronic publication of two journals on Latin American social medicine.

\section{REFERENCIAS}

1. Waitzkin H, Iriart C, Estrada A, Lamadrid S. Social medicine in Latin America - productivity and dangers facing the major national groups. Lancet 2001;358: 315-323.

2. Waitzkin $\mathrm{H}$, Iriart C, Estrada A, Lamadrid S. Social medicine then and now: lessons from Latin America. Am J Public Health 2001;91:1592-1601.

3. Nunes ED. Trayectoria de la medicina social en América Latina: elementos para su configuración. En: Franco S, Nunes E, Breilh J, Laurell AC, eds. Debates en Medicina Social. Quito: Organización Panamericana de la Salud/ Asociación Latinoamericana de Medicina Social; 1991.

4. Donnangelo MCF. Salud y sociedad. Guadalajara, México: Editorial de la Universidad de Guadalajara; 1994.

5. Iriart C, Nervi L, Olivier B, Testa M. Tecnoburocracia sanitaria. Ciencia, ideología, y profesionalización. Buenos Aires: Lugar Editorial; 1994

6. Menéndez E. Salud pública: sector estatal, ciencia aplicada o ideología de lo posible. En: Organización Panamericana de la Salud. La crisis de la salud pública: reflexiones para el debate. Washigton, D.C.: OPS; 1992. (Publicación Científica No. 540). Pp. 103-122.

7. Testa M. Salud pública y atención primaria de salud: una evaluación crítica. En: Organización Panamericana de la Salud. La Crisis de la salud pública: reflexiones para el debate. Washington, D.C.: OPS; 1992. (Publicación Científica No. 540). Pp. 205-229.

8. Laurell AC. La salud y la enfermedad como proceso social. Revista Latinoamericana de Salud (México, D.F.) 1982 abril $2: 7-25$.

9. Behm Rosas H. Mortalidad infantil y nivel de vida. Santiago de Chile: Ediciones de la Universidad de Chile; 1962.

10. Breilh J, Granda E. Acumulación económica y salud-enfermedad: la morbimortalidad en la era del petróleo. Revista Salud y Trabajo (Quito) 1982 junio;2:2-29.
11. García JC. La educación médica en América Latina. Washington, D.C.: Organización Panamericana de la Salud; 1972. (Publicación Científica No. 255).

12. García JC. La investigación en el campo de la salud en once países de la América Latina. Washington, D.C.: Organización Panamericana de la Salud; 1982.

13. García JC. La mortalidad de la niñez temprana según clases sociales. Medellín, Colombia: Universidad Pontífica Bolivariana; 1979.

14. Franco S, Nunes E, Breilh J, Laurell AC, eds. Debates en Medicina Social. Quito: Organización Panamericana de la Salud/ Asociación Latinoamericana de Medicina Social; 1991.

15. Breilh J. Epidemiología: economía, medicina y política. $3^{\mathrm{a}}$. ed. México, D.F.: Fontamara; 1989

16. Breilh J. Nuevos conceptos y técnicas de investigación. $3^{\text {a }}$. ed. Quito: Centro de Estudios y Asesoría en Salud; 1997.

17. Laurell AC, Blanco Gil J, Machetto T, Palomo J, Pérez Rulfo C, Ruí de Chávez M, et al. Enfermedad y desarrollo: análisis sociológico de la morbilidad en dos pueblos mexicanos. Revista Mexicana de Ciencias Políticas (México) 1976;84:131158.

18. Laurell AC, Noriega M, López O, Ríos $\mathrm{V}$. La experiencia obrera como fuente de conocimiento: confrontación de resultados de la encuesta colectiva e individual. Cuadernos Médico Sociales 1990; 51:5-26.

19. Almeida N. La ciencia tímida: ensayos de deconstrucción de la epidemiología. Buenos Aires: Lugar Editorial; 2000.

20. Franco $S$, Nunes ED. Presentación. En: Franco S, Nunes E, Breilh J, Laurell AC, eds. Debates en Medicina Social. Quito: Organización Panamericana de la Salud/ Asociación Latinoamericana de Medicina Social; 1991. Pp. 7-16.

21. Victoria C, Barros F, Vaughan P. Epidemiología de la desigualdad. Washington, D.C.: Organización Panamericana de la Salud; 1992. (Serie PALTEX No. 27).
22. Breilh J. El género entre fuegos: inequidad y esperanza. Quito: Centro de Estudios y Asesoría en Salud; 1996.

23. Quevedo E. La articulación del conocimiento básico y social en la formación del personal de salud: una mirada desde la historia. En: Rodríguez MI, ed. Lo biológico y lo social. Washington: Organización Panamericana de la Salud; 1994. (Serie Desarrollo de los Recursos Humanos No. 101). Pp. 13-34.

24. Romero A. El desarrollo histórico de la epidemiología en América Latina. En: Anais do I Congreso Brasileiro de Epidemiologia; 1990, Campinas.

25. Gramsci A. Quaderni del carcere. Edición crítica de Valentino Gerratana. Turín: Einaudi; 1975.

26. Costa NR. Transición y movimientos sociales: contribuciones al debate de la reforma sanitaria. Cuadernos Médico Sociales 1988;44:51-61.

27. Breilh J. Componente de metodología: la construcción del pensamiento en medicina social. En: Franco S, Nunes E, Breilh J, Laurell AC, eds. Debates en Medicina Social. Quito: Organización Panamericana de la Salud; 1991.

28. Laurell AC. Social analysis of collective health in Latin America. Soc Sci Med 1989;28:1183-1191.

29. Franco AS. La cuestión de la causalidad en medicina. En: Grupo de Trabajo. Desarrollo de la Medicina Social en America Latina, OPS-ALAMES. México, D.F.: Organización Panamericana de la Salud; 1989.

30. Rosen G. De la policía médica a la medicina social. México, D.F.: Siglo XXI; 1985.

31. Franco AS. Tendencias de la medicina social en América Latina. Salud y cambio (Santiago de Chile) 1990;2:4-16.

32. Laurell AC, Noriega M. Proceso de trabajo y salud en SICARTSA. Cuadernos Médico Sociales 1987;40:5-24.

33. Pitta A. Hospital, dor e morte como oficio. $4^{\mathrm{a}}$. ed. São Paulo: Editora Hucitec; 1999. 
34. Campaña A. Salud mental: conciencia vs. seducción por la locura. Quito: Ediciones CEAS; 1995.

35. Goldemberg P. Condiciones de vida y salud en los años 80: reproducción social como categoría de análisis. Cuadernos Médico Sociales 1991;57:35-54.

36. Breilh J. Género, poder y salud. Quito: Centro de Estudios y Asesoría en Salud; 1993.

37. Testa M. Pensar en salud. Buenos Aires: Lugar Editorial; 1993.

38. Testa M. Pensamiento estratégico y lógica de programación. Buenos Aires: Lugar Editorial; 1996.

39. Laurell AC, López Arellano O. Market commodities and poor relief: The World Bank proposal for health. Int J Health Serv 1996;26:1-18

40. Stocker $K$, Waitzkin $H$, Iriart $C$. The exportation of managed care to Latin America. N Engl J Med 1999;340:11311136.

41. Iriart C, Merhy E, Waitzkin H. Managed care in Latin America: the new common sense in health policy reform. Soc Sci Med 2001;52:1243-1253.

42. Merhy E, Onocko R. Agir em saúde: um desafio para o público. São Paulo: Editora Hucitec/Buenos Aires: Lugar Editorial; 1997.

43. Iriart C, Merhy E, Waitzkin H. Atención gerenciada en América Latina: transnacionalización del sector salud en el contexto de la reforma. Cad Saude Publica 2000;16:95-105.

44. Iriart C. La reforma del sector salud en Argentina: de la salud como derecho social a bien público a responsabilidad individual y bien de mercado. En: Centro de Estudios y Asesoría en Salud. Reforma en salud: lo privado o lo solidario. Quito: CEAS; 1997.
45. Breilh J. Reforma: democracia profunda, no retroceso neoliberal. En: Centro de Estudios y Asesoría en Salud. Reforma en salud: lo privado o lo solidario. Quito: CEAS; 1997.

46. Merhy E, Iriart C, Waitzkin H. Atenção gerenciada: da micro-decisão corporativa à micro-decisão administrativa, um caminho igualmente privatizante? En: Bursmester H, ed. Managed care: alternativas de gestão em saúde. São Paulo: Editora PROAHSA/Editora Fundação Getulio Vargas; 1998.

47. Laurell AC, Ortega M. The free trade agreement and the Mexican health sector. Int J Health Serv 1992;22:331-337.

48. Laurell AC, Wences M. Do poverty programs alleviate poverty? The case of the Mexican National Solidarity Program. Int J Health Serv 1994;24:381-401.

49. De Oliveira Cecilio LC ed. Inventando a mundaça. São Paulo: Editora Hucitec; 1994.

50. Franco T, Bueno W, Merhy E. O acolhimento e os processos de trabalho em saúde: o caso de Betim, Minas Gerais Brasil. Cad Saude Publica 1999;15:345-53.

51. Campos GWS. Reforma da reforma. Repensando a saúde. São Paulo: Editora Hucitec; 1992

52. Campos CR, Malta DC, Teixeira dos Reis A, dos Santos A, Merhy EE. Sistema Único de Saúde em Belo Horizonte. Reescrevendo o público. São Paulo: Xamá Editora; 1998.

53. Iriart C, Merhy E, Waitzkin H. La atención gerenciada en América Latina: su papel en la reforma de los sistemas de salud. Buenos Aires: Instituto de Estudios y Formación/Central de Trabajadores Argentinos; 1999

54. Colectivo CEAS. Mujer, trabajo y salud. Quito: Ediciones CEAS; 1994.
55. Betancourt O. La salud y el trabajo. Quito: Ediciones CEAS/Organización Panamericana de la Salud; 1995.

56. Breilh J, Granda E, Campaña A, Betancourt O. Ciudad y muerte infantil. Quito: Ediciones CEAS; 1983.

57. Laurell AC, Noriega M, Martínez S, Villegas J. Participatory research on workers health. Soc Sci Med 1992;34: 603-613.

58. Montecino S. Madres niñas, madres solas, continuidad o cambio cultural? Salud y Cambio (Santiago de Chile) 1993;4: 6-8.

59. Franco AS. Violencia y salud en Colombia. Rev Panam Salud Publica 1997;1:93103.

60. Franco S. International dimensions of Colombian violence. Int J Health Serv 2000;30:163-185.

61. Estrada A, Hering M, Donoso A. Familia, género y terapia: una experiencia de terapia familiar sistémica. Santiago de Chile: Ediciones CODEPU; 1997.

62. Martín-Baró I. Writings for a liberation psychology. Cambridge, MA: Harvard University Press; 1994.

63. Martín-Baró I. La violencia en Centroamérica: una visión psicosocial. Salud, Problema y Debate (Buenos Aires) 1990; 2:53-66.

64. Stolkiner A. Tiempos "posmodernos": ajuste y salud mental. En: Cohen $\mathrm{H}$, de Santos B, Fiasché A, Galende E, Saidón $\mathrm{O}$, Stolkiner A, et al., eds. Políticas en salud mental. Buenos Aires: Lugar Editorial; 1994.

65. Ad Hoc Working Group for Critical Appraisal of the Medical Literature. A proposal for more informative abstracts of clinical articles. Ann Intern Med 1990; 113:69-76.

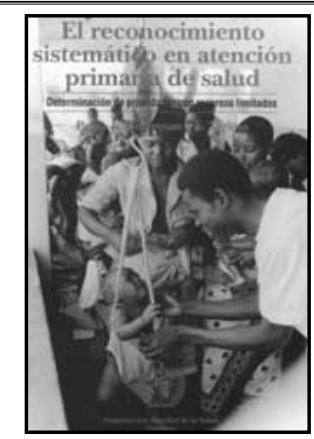

$1996 \cdot 214 \mathrm{pp}$

ISBN $924354473 \mathrm{X}$

Código: WHO 11•

Precio: US\$27.00/

US\$ 21.00 en América

Latina y el Caribe

\section{El reconocimiento sistemático en atención primaria de salud: determinación de prioridades con recursos limitados}

En esta publicación se examina cómo se integra el reconocimiento sistemático en un servicio basado en la atención primaria de salud, con objeto de utilizar los recursos de una manera más equitativa, eficiente y eficaz. Se recomiendan criterios para facilitar a las autoridades la decisión de incluir o no el reconocimiento sistemático en los planes para la prevención de determinado trastorno de salud y se exponen en líneas generales los principios aplicables en la planificación y prestación de servicios que comprenden el reconocimiento sistemático. Se ofrecen ejemplos de cómo éste puede usarse para aclarar la aplicación del método recomendado y se indican posibles opciones. Las recomendaciones formuladas en esta obra no son preceptivas; su objeto es más bien alentar a las autoridades nacionales a establecer directrices que sean apropiadas para el ámbito local sobre prácticas clínicas que conduzcan al mejoramiento de la salud y faciliten el acceso de toda la población a servicios preventivos y curativos. 